\title{
Prehospital Care and 24-hour Crash Injury Mortality Among Road Traffic Crash Victims in Addis Ababa, Ethiopia
}

\author{
Zuriyash Mengistu ${ }^{1}$, Ahmed Ali ${ }^{2}$, Teferi Abegaz ${ }^{2}$ \\ ${ }^{1}$ School of Nursing and Midwifery, College of Health Sciences, Addis Ababa University, Addis Ababa, Ethiopia \\ ${ }^{2}$ School of Public Health, College of Health Sciences, Addis Ababa University, Addis Ababa, Ethiopia \\ Email address: \\ zuriyashaau@yahoo.com (Z. Mengistu), ahmedaa5050@yahoo.com (A. Ali), teferiabegaz@gmail.com (T. Abegaz) \\ ${ }^{*}$ Corresponding author
}

To cite this article:

Zuriyash Mengistu, Ahmed Ali, Teferi Abegaz. Prehospital Care and 24-hour Crash Injury Mortality Among Road Traffic Crash Victims in Addis Ababa, Ethiopia. Science Journal of Public Health. Vol. 9, No. 1, 2021, pp. 23-29. doi: 10.11648/j.sjph.20210901.13

Received: December 1, 2020; Accepted: December 17, 2020; Published: February 2, 2021

\begin{abstract}
Road traffic crash injuries are critical public health problems that demand on time post crash response to deliver pre-hospital care. Ethiopia is one of the African countries with the worst pre-hospital care delivery platform. This study describes the current practice of pre-hospital service provided for crash injured individuals. A cross-sectional study was conducted among 430 crash victims attending public hospitals in Addis Ababa. Participants were selected by random sampling upon their arrival to the selected hospitals from January 2019 to August 2020. The study described the data using the percentage, median, and interquartile range. The study used binary logistic regression to assess the relationship between prehospital care provided and 24-hour crash victim mortality. The median scene time was 20 minutes. More than half of respondents transported to hospital greater than 60 minutes. Among the 430 injured individuals, the fatal crash occurred on 21 (4.9\%) individuals. The crash injured individuals received pre-hospital care involving wound care, resuscitations with IV (Intravenous) fluid, and immobilization. Using the binary logistic regression model crash injured patients who did not receive ambulance service were three times more chance of mortality during a 24-hour crash event $((\mathrm{P}$-value $=0.002, \mathrm{AOR}=3.1(95 \% \mathrm{CI}$ $1.5-6.4)$. This study showed that $33.3 \%$ of crash-injured individuals received pre-hospital care. The majority of participants arrived at tertiary level trauma hospital with time greater than platinium 10 minutes scene time and golden an hour transport time. Being male, young, and not receiving pre-hospital care were significantly related to early crash injury mortality. A suitable basic and advanced life support strategy should be designed and implemented to enhance timely crash injury care.
\end{abstract}

Keywords: Crash Injury, Prehospital Injury Care, Road Traffic Crash Injury Mortality

\section{Introduction}

Road traffic crash injury is a public health problem resulting from the physical force generated from collusion act upon the vulnerable human body. Particularly, the prehospital arena is considered by many to be the most challenging because of its predisposition for adverse factors that require the highest quality care framework within the context of the pre-hospital environment [1].

A crash victim with major injury critically needs timely pre-hospital care, subsequent transportation, and timely access to trauma center care to reduce the preventable death. Pre-hospital care is organized around different schemes worldwide [1, 2]. Efficient emergency care during pre- hospital time integrated with hospital trauma care services plays an essential role to limit crash consequences [2].

The number of potentially preventable death remain high in which improving trauma center access will be critical to achieving "Zero Preventable Deaths after Injury." [3, 4]. Once a crash injury occurs, the person requires fast and adequate medical care that is often viewed as a "chain of survival," stretching from the site of injury in the field to the emergency department, to the operating room, to the intensive care unit, and beyond to the rehabilitation center. The care of the same problem may vary depending on the point of care [2]. According to the World Health Organization (WHO), many victims may needlessly die at the scene or during the first few hours following injury due to poor pre-hospital care [5]. The study revealed that for young male victims, prehospital trauma 
systems reduce mortality by $25 \%$ in developing countries [6].

Studies showed that road traffic injured individuals were transported commonly by taxies, and by non-Emergency Medical Service (EMS) [7-11]. A few who were transported by ambulance had a severe injury when compared with those that arrive at health care systems in other modes [12]. Moreover, victims transported by ambulance required advanced procedures like intubation, chest tube insertion, and blood transfusion at the emergency department. [pre hospital transport [11]. Moreover, careful handling and transporting of victims using fast and adequate mode of transportation with appropriate lifesaving assistants are vital [11-12]. Unfortunately, studies indicated that the majority of the victims were extracted by bystanders from the scene of the accident, and most used commercial vehicle to go to a health institution [12].

Golden an hour to arrive at a definitive trauma care center along with trauma care is considered as a cornerstone of trauma systems. Small differences may always play a crucial role in determining the outcome for all trauma patients. But studies showed that the overall mean arrival time for all patients was most commonly greater than the golden hour [11, 12]. On the other hand, it was reported that the majority of the victims arrived at the health institute within the golden hour $[12,13]$. Similar to golden an hour to arrive at a definitive trauma care center, the "platinum 10 minutes" has arisen in pre-hospital lore to declare that seriously injured patients should have no more than 10 minutes of scene-time stabilization before transport to definitive care at a trauma center [14].

Exceptionally, there has been a challenge in identifying and defining which patients have time-dependent injuries where pre-hospital time may have a direct impact on outcome [15]. Hence, the golden hour may not equally apply to a crash victim who has a rupture of the left ventricle, the difference between life and death may be a golden 5 minutes [14]. Correspondingly, studies revealed that survival of victim after major trauma is associated with careful assessment for the injury severity, efficient initial care and directly taken to tertiary care trauma center without rushing the victims to another hospital along with distinguishing time-sensitive causality $[16,17]$.

Studies also reported that there were high pre-hospital fatalities for road traffic crash injured individuals [8-10, 18]. In particular, Ethiopia is one of the African countries with the worst road traffic injury record which needs investigation to provide appropriate corrective measures [8-10]. Pre-hospital care services in Ethiopia have not been treated as a priority and are characterized by inadequate coordination among actors. For instance, in Addis Ababa, only 20.3\% of patients arrived at hospitals by ambulance services, and the rest managed by bystanders and public transportation services [19].

Therefore, it is timely and important to examine the prehospital care of road traffic injury for informed decision making and management. Thus this study attempts to assess the extent of pre-hospital care and injury severity among road traffic crash injury victims in Addis Ababa, Ethiopia.

\section{Methods and Materials}

\subsection{Study Area and Period}

An Institutional based cross-sectional study was conducted to assess the extent of pre-hospital care provision for road traffic crash injured victims. The study was conducted in three Addis Ababa city public hospitals' emergency department triage which includes, AaBET (Addis Ababa Burn, Emergency and Trauma) Hospital, Tikur Anbessa Specialized Teaching Hospital, and Minilik Hospital in Addis Ababa City Administration during the period of January 2019 to August 2020. These hospitals currently provide tertiary level emergency trauma care services in Addis Ababa.

\subsection{Study Design}

An institution-based cross-sectional study design was employed.

\subsection{Population}

\subsubsection{Source Population}

All crash injured patients arrived at tertiary level trauma care hospitals in this study area during the study period.

\subsubsection{Study Population}

All crash injured patient who arrived at tertiary level trauma care hospitals in the study period and fulfill the inclusion criteria during the study period.

Inclusion Criteria

All road traffic crash victims aged 15 years and above transported to the selected three hospitals were included in the study.

Exclusion Criteria

Road crash victims who were brought to the hospital deadon-arrival, incapable to respond, and did not have a surrogate (bystanders or family members) during the crash were excluded from the study.

\subsection{Sample Size Determination}

Sample size was computed by using a single population proportion formula. The following assumptions were used for sample size determination using a crude estimate proportion of emergency medical service ambulance care $(22.61 \%)$ as reported by other study [10], with $95 \%$ confidence level of discrepancy rate of $5 \%$, a design effect of 1.5 and adding a $10 \%$ contingency for non-response rate.

$$
\mathrm{n}=\frac{\left(\mathrm{Z} \alpha-\frac{1}{2}\right)^{2} \times p(1-p)}{d^{2}}
$$

$272(1.5)+27=435$ sample was computed.

\subsection{Sampling Technique}

All public hospitals within the city were included to make the data representative.

The three hospitals were selected by simple random sampling from five tertiary level trauma care hospitals in the 
city. After the resuscitation of victim, study participant who experienced crash injury in Addis Ababa road during the study period was selected at emergency department triage of each selected hospital.

\subsection{Variables}

\subsubsection{Dependent Variable}

A 24-hour crash victims' mortality.

\subsubsection{Independent Variable}

i. Socio demographic characteristics; age, ethnicity, sex and educational level.

ii. injury site and severity.

iii pre hospital time and pre hospital care.

\subsection{Operational Definition}

Pre hospital care was an emergency care given for crash victims starting from scene to hospital arrival. On-scene time was the time spent at the scene for treatment and loading of the patient. Transport time was the time from departure from trauma scene to arrival at the hospital for definitive care. Total pre-hospital time was defined as the total time spent between the emergency call and the arrival at the hospital.

\subsection{Data Collection Method and Quality Assurance}

In this study emergency physician and nurses collected the data using a structured and pretested questionnaire. The check list was used to review the injured patient medical record chart. Structured questionnaire that translated to Amharic and then to English by language expert was used for interview. The data was collected from eligible individual after getting informed consent at emergency triage by a face to face interview and reviewing the victim's clinical data record chart. Training was given for data collectors and supervisors. Data were checked for completeness, missing values, inconsistency, both by the supervisors and by principal investigator on a daily basis. To assure trustworthiness of the data the principal investigator was work with data collectors to minimize inter-observer bias. Correctly completed questioner was collected from data collectors.

\subsection{Data Processing and Analysis}

The collected data was entered, cleaned and analyzed using SPSS for windows version 24. Frequency distributions and percentages, median, and interquartile range were computed for descriptive statistics. A binary logistic regression was used to identify association between independent and the outcome variable finally, multiple logistic regressions was used to identify more commonly associated risk factors. The degree of association between dependent and independent variables examined using odds ratio with $95 \% \mathrm{CI}$ and $\mathrm{p}<0.05$.

\subsection{Ethical Considerations}

The research project was approved by the Institutional
Review Board (IRB) of the College of Health Sciences, Addis Ababa University. Ethical clearance protocol number: 036/17/SpH was received, before the commencement of the study. Permission was obtained from each hospital setting. Both injured persons and/or surrogates received detailed information on the purpose of the study. Then data were collected after they were asked for consent to be part of this study. The injured person's identity data was not exposed and confidentiality of information about road traffic injured patient was kept.

\section{Results}

\subsection{Socio-demographic Characteristics of the Study Population}

A total of 430 injured victims were consented and agreed to participate in this study with a $99 \%$ response rate. Males outnumbered females with a sex ratio of $2.5: 1$. The median age of injured persons was 28 years with an interquartile range from 22 to 40 years. The age group, between 15 - and 34-year accounting for 278 (64.7\%) (Table 1). The commonest ethnic group was Oromo with a frequency of 195 (45.3\%) followed by Amhara 168 (39.1\%). Concerning educational status, $210(48.8 \%)$ attended secondary school followed by $86(20 \%)$ primary school. A higher proportion of injured individuals was passenger accounting for 204 (47.5\%), followed by pedesterians $187(43.5 \%)$.

Table 1. Socio-demographic characteristics of road traffic crash injured individuals transported from the scene to Emergency medical triage unit, Addis Ababa, Ethiopia, $2020(n=430)$.

\begin{tabular}{|c|c|c|}
\hline Characteristics & Frequency & Percentage $(\%)$ \\
\hline \multicolumn{3}{|l|}{ Sex } \\
\hline Male & 306 & 71.2 \\
\hline Female & 124 & 28.8 \\
\hline \multicolumn{3}{|l|}{ Age } \\
\hline $15-34$ & 278 & 64.7 \\
\hline $35-54$ & 111 & 25.8 \\
\hline$>54$ & 41 & 9.5 \\
\hline \multicolumn{3}{|l|}{ Ethnicity } \\
\hline Oromo & 195 & 45.3 \\
\hline Amhara & 168 & 39.2 \\
\hline Gurage & 23 & 5.3 \\
\hline Tigray & 19 & 4.4 \\
\hline$*$ Others & 25 & 5.8 \\
\hline \multicolumn{3}{|l|}{ Educational status: } \\
\hline Illiterate & 73 & 17.0 \\
\hline Primary school & 86 & 20.0 \\
\hline Secondary school & 210 & 48.8 \\
\hline Higher education & 61 & 14.2 \\
\hline \multicolumn{3}{|c|}{ Type of Road participation: } \\
\hline Pedestrians & 187 & 43.5 \\
\hline Passenger & 204 & 47.5 \\
\hline Driver & 39 & 9.0 \\
\hline
\end{tabular}

*Others (Silte, Hadya, Welayta, Somali, Sidama) 
Table 2. Crash injury characteristics among injured individuals transported from scene to hospital at in Addis Ababa, Ethiopia, 2020 (n=430).

\begin{tabular}{lll}
\hline Site of injury & Frequency & Percent (\%) \\
\hline Head-neck / face & 79 & 18.4 \\
Multiple injury sites & 113 & 26.3 \\
Chest & 13 & 3.0 \\
Abdomen- pelvic & 18 & 4.2 \\
Extremity & 207 & 48.1 \\
*NISS & & \\
NISS $<15$ & 297 & 69.1 \\
NISS $16-24$ & 77 & 17.9 \\
NISS $\geq 25$ & 56 & 13.0 \\
24 Hour injury fatality: & & 4.9 \\
Died & 24 & 95,1 \\
Alive & 409 & \\
\hline
\end{tabular}

*NISS (New Injury Severity Score.

\subsection{Crash Injury Characteristics}

Almost half of the participants accounting for 207 (48.1\%) had extremity injury followed by multiple injury sites, 113 (26.3\%). The majority of the individual had a New Injury Severity Score less than fifty, 297 (69.1\%). A total of 21 $(4.9 \%)$ road traffic crash victims died within 24 hours of crash occurrence (Table 2).

\subsection{Injury Site and Survival Status of the Injured Patient}

Among 71 head/neck/face injured patients, accounting for $15(21.1 \%)$ died within 24 hours of the crash event. Among 109 victims with multiple site injuries, accounting for 4 (3.7\%) died within 24-hour crash injury occurrence. Nearly half of crash victims had extremity injury without any death (See Figure 1).

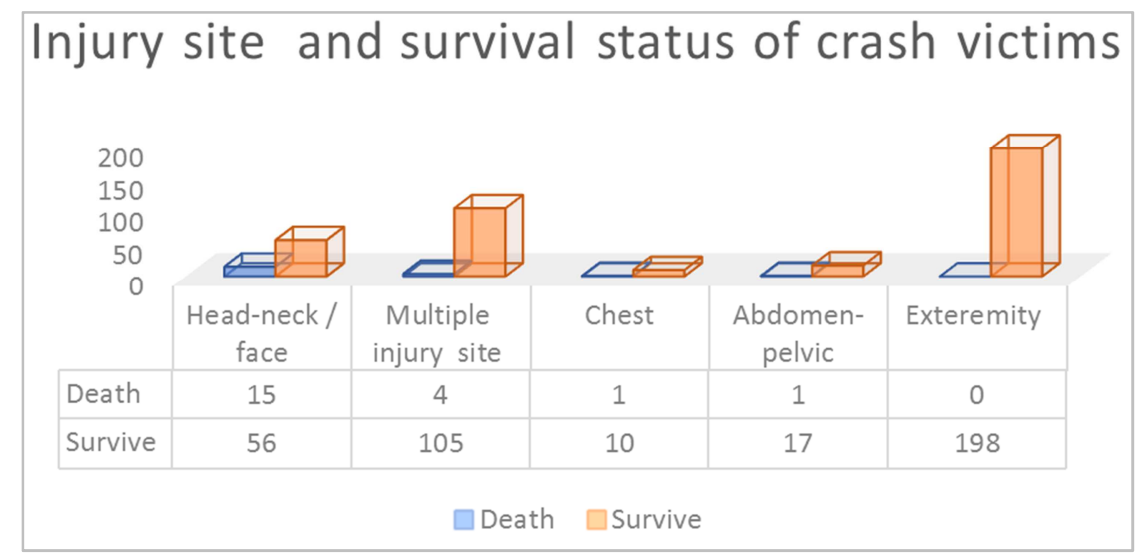

Figure 1. Site of injury and survival status of crash victims among road traffic crash injury at Addis Ababa City, Ethiopia 2020 (n=430).

\subsection{Prehospital Care Provided for Cras Victims}

Among the total of 430 injured individuals, 142 (33.3\%) received pre-hospital care. The majority of crash victims were transported to definitive trauma care hospitals by Taxi, $216(50.2 \%)$.

The main pre-hospital care provided were wound care and dressing, accountibg for 70 (16.3\%). Resuscitation /IV fluid/, splint, immobilization resuscitation /IV fluid/, splint, immobilization were provided for 51 (19.3\%) individuals (Table 3).

The median scene time was 20 minutes with an interquartile range of 14 to 29 minutes. More than half of the crash victims stayed on the scene for more than 20 minutes. The median transporting time was 41 minutes with an interquartile range of 25 to 60 minutes. The commonest transport time was 30 to 60 minutes. More than half of participants accounting for $226(52.6 \%)$ transported longer than 60 minutes.

\subsection{The associated Factor of 24-hour Crash Victims Mortality}

For those variables that had a significant association to the dependent variable, a P-value of less than 0.2 was entered into multiple logistic regressions.

Crash victims who were males were two times more likely to die within 24 - hours of a crash event than females (Pvalue $=0.03, \mathrm{AOR}=2.1(95 \% \mathrm{CI} 1.04-4.4))($ Table 4).

Those crash victims in the age group 34 to 54 years were 5.6 times more likely to die within 24-hours of crash occurrence than age group greater than 54 years (Pvalue $=0.001, \mathrm{AOR}=5.6$ (95\%CI 2.1. -15.2$)$.

A crash injured patient who did not receive pre-hospital 
care were 3.1 times more likely to die during a 24-hour of the

crash event $((\mathrm{P}$-value $=0.002, \mathrm{AOR}=3.1(95 \% \mathrm{CI} 1.5-6.4)$.

Table 3. Pre-Hospital Care provided for crash victims among mobile person in trip Addis Ababa, Ethiopia, 2020 ( $n=430)$.

\begin{tabular}{lll}
\hline Characteristics & Frequency & Percentage (\%) \\
\hline Mode of transportation to hospital: & & 33.3 \\
Ambulance & 143 & 50.2 \\
Taxi & 216 & 16.5 \\
Private & 71 & 16.3 \\
Type of care provided: & & 11.9 \\
Wound care and dressing & 70 & 3.0 \\
Resuscitation/IV fluid/, splint, immobilization & 51 & 2.3 \\
Neck collar/lumbar brace_immobilization & 13 & 66.5 \\
Stop bleeding, wound care+ + immobilization & 10 & \\
Undefine & 286 & 2.8 \\
Prehospital time & & 43.0 \\
Scene time: & 12 & 54.2 \\
On-scene time $<10$ minutes & 185 & \\
On-scene time $10-19$ minutes & 233 & 26.0 \\
On-scene time $>=20$ minutes & & 42.8 \\
Transport time: & 112 & 31.2 \\
$<30$ minutes & 184 & \\
30 - 60 minutes & 134 & 47.4 \\
$>60$ minutes & & 52.6 \\
Total pre-hospital time & 204 & \\
$<=60$ minutes & 226 & \\
$>60$ minutes & &
\end{tabular}

Table 4. Logistic Regression Analyses for factors associated with 24-hour post-crash injury mortality among crash victims Addis Ababa hospitals, Ethiopia, $2020(n=430)$.

\begin{tabular}{|c|c|c|c|c|}
\hline \multirow{2}{*}{ Variables } & \multicolumn{2}{|c|}{ Crash mortality } & \multirow{2}{*}{$\begin{array}{l}\text { Crude odds ratio (95\% } \\
\text { Confidence Interval) }\end{array}$} & \multirow{2}{*}{$\begin{array}{l}\text { Adjusted odds ratio (95\% } \\
\text { Confidence Interval) }\end{array}$} \\
\hline & Yes n $(\%)$ & No n $(\%)$ & & \\
\hline \multicolumn{5}{|l|}{ Sex: } \\
\hline Female & $5(4.0)$ & $119(96.0)$ & Ref & \\
\hline Male & $16(5.2)$ & $290(94.8)$ & $18.1(10.9-29.9)$ & $2.1(1.0-4.4)$ \\
\hline \multicolumn{5}{|l|}{ Age in years: } \\
\hline $15-34$ & $15(5.4)$ & $263(94.6)$ & $17.5(10.4-29.5)$ & $4.9(2.3-10.2)$ \\
\hline$>54$ & $0(0.0)$ & $41(100.0)$ & Ref & \\
\hline Pre hospital care: & $0(0.0)$ & $70(100.0)$ & Ref & \\
\hline Wound care, dressing & $2(3.9)$ & $49(96.1)$ & $24.5(5.9-99.7)$ & $3.5(0.8-16.4)$ \\
\hline Neck collar/back brace/splint immobilization & $3(23.1)$ & $10(76.9)$ & $3.3(0.9-12.1)$ & $0.5(0.1-2.1)$ \\
\hline Resuscitation/IV fluid/immobilization & $1(10.0)$ & $10(90.0)$ & $9.0(1.1-71.0)$ & $2.2(0.3-19.5)$ \\
\hline Stop bleeding/wound care/immobilization Undefined & $15(5.2)$ & $271(94.8)$ & $18.1(10.7-30.4)$ & $3.1(1.5-6.4)$ \\
\hline
\end{tabular}

\section{Discussion}

The findings of this study showed that low basic life support and the non existence of advanced life support prehospital care for road traffic injury victims, which is almost similar to the previous studies in Ethiopia [7-11]. The low pre-hospital care may be explained by minimum awareness of the importance of ambulance care and limited access to ambulance care. In this study, the median transporting time was 41 minutes with an interquartile range of 25 to 60 minutes. This pre-hospital time is higher than the study conducted in a Scandinavian country [13]. But lower than the previous study conducted in Ethiopia [7]. Our study focused on Addis Ababa city road injury victims with probably shorter distance traveling than the studies that included subjects outside Addis Ababa. More than half of the victims who used a taxi as a mode of transportation transported longer than 60 minutes. This is in agreement with the previous study conducted in the same area in Addis Ababa City [8].

The majority of the victims in this study were in the younger and most productive age group of 15 to 34 years old, followed by 35-54 years. This is almost similar to other previous studies [7-10]. This may be attributable to the fact that this is the age group that tends to overspend their time in outdoor activities. This study also found that males had a higher risk of injury than females with a male to female ratio of 2.5 to 1 . This is almost similar to previous reports [7-10]. This may be explained by the fact that the males were more 
involved in outdoor activities. Therefore, this study indicated that the majority of road traffic crash victims were young males in their most reproductive and productive years.

This study also showed that almost half (48.1\%) of road users on trip incurred extremity crash injury. This is slightly lower than the previous studies, (53.8\% in Ethiopia [8]. 56.4\% in Iran [9]. This may be due to that the previous studies included rural crash injuries that differ in crash involved vehicle type and speed. In cities here had regulations specified for low-speed limits whereas in rural settings mostly heavy vehicles travel long distances with high speed. The study found out pre-hospital fatality of about $4.9 \%$, lower than the study in Addis Ababa reported 7.4\% [7].

The study showed that early crash mortality was significantly higher in males than in females. This finding was similar to another study conducted in Japan [18]. Lack of receiving prehospital care was significantly associated with early mortality. This finding was inconsistent with the previous study [7].

The study may have some limitations: First, the data was collected where the crash injury victims have been in physical and psychological stress situations that can limit recalling the exact measure of scene and transport time values. Second, the study design is cross-sectional so that could not determine the effect of specific hospital care for early crash mortality. Thirdly, a limited number of crash injured individuals might have arrived at hospitals. Lastly, collecting data at the time of hospital arrival may not be inclusive to collect the overall situation of scene time and transport time pre-hospital characteristics of crash injured victims.

\section{Conclusion and Recommendation}

In conclusion, this study has shown that $33.3 \%$ of crash injured individual received pre-hospital care during transport from the scene to a definitive care provider hospital. Being male, younger age, and not receiving pre-hospital care was significantly related to early crash victim mortality. Delivering appropriate information regarding crash safety for travelers is essential to reduce the road traffic crash early mortality. Accordingly, suitable basic and advanced life support strategies should be designed and implemented for acceptable crash injury response.

For better hospital care access, decreasing the time spent in the pre-hospital setting, using an ambulance service, increasing public awareness about pre hospital care within the platinium 10 minutes scene time and golden an hour prehospital time should be areas of emphasis.

\section{List of Abbreviations}

AaBET - Addis Ababa Burn, Emergency and Trauma, AU - African Union, EMS - Emergency Medical Service, IRB Institutional Review Board, IV- Intravenous, NISS- New Injury Severity Score, RTI - Road Traffic Injury, RTIs Road Traffic Injuries, IQR Inter Quartile Range, UNECA -
United Nations Economic Commission for Africa

\section{Ethical Approval and Consent to Participants}

Ethical approval was obtained from the institutional review board of Addis Ababa University College of health science school of Public Health, Permission was obtained from selected Addis Ababa Hospitals. All respondents were informed about the objective of the study and their free choice to participate or withdraw whenever they wished during the data collection period. Confidentiality of information was maintained.

\section{Availability of Data and Materials}

The data is presented along with the manuscript.

\section{Consent for Publication}

Not applicable.

\section{Competing Interest}

The authors declare that there is no conflict of interest regarding the publication of this paper.

\section{Authors' Contributions}

ZM was involved in conception, design, analysis, interpretation, report, and manuscript writing. AA and TA were involved in supervising, reviewing, and editing the manuscript.

\section{Acknowledgements}

The authors' gratitude goes to Addis Ababa University for financial support. The authors extend their gratitude to study subjects for their participation and willingness to be involved in the study.

\section{References}

[1] Schagen I, Wegman F. Houtenbos M. Global road safety: promising routes to further improvements. Review paper. SWOV Institute for Road Safety Research. The Netherlands. 2013. www.swov.nl.

[2] Health Information and Quality Authority. Review of prehospital emergency care services to ensure high quality in the assessment, diagnosis, clinical management, and transporting of acutely ill patients to appropriate health facilities. The UK. 2014. www.hike.ie.

[3] Hashmi et al. Trauma Center Access and Pre-Hospital Death J Am Coll Surg. 2018. https://doi.org/10.1016/j.jamcollsurg.2018.09.015 ISSN 1072$7515 / 18$. 
[4] Oliver G. J, Walter D. P, Redmond A. D. G. J. Are prehospital deaths from trauma and accidental injury preventable? A direct historical comparison to assess what has changed in two decades. Injury, Int. J. Care Injured. (2017; 48: 978-984. www.e lsevier.com/loca te/ injury.

[5] A 5-year strategy to address road traffic injuries globally Report. Geneva, Switzerland. World Health Organization $2001 \mathrm{http}: / / \mathrm{www}$. who.int/violence_injury_prevention.

[6] Henry J. A, Reingold A L. Prehospital trauma systems reduce mortality in developing countries: A systematic review and meta-analysis. J Trauma Acute Care Surg 2012; 73 (1): 261-8. DOI: 10.1097/TA.0b013e31824bde1e.

[7] Yehune Meskere, Muluwork Tefera Dinberu, Aklilu Azazh. Patterns and determinants of pre-hospital care among trauma patients treated in Tikur Anbessa Specialized Hospital, emergency department. Ethiop Med J. 2015; 53 (3): 141-9.

[8] Seife H, Teffera E. Epidemiology of road traffic accidents: A Prospective study At a Tertiary University Hospital in Addis Ababa Ethiopia. East \& Central African Journal of Surgery. 2015; 20 (1): 3-9.

[9] Paravar M, Hosseinpour M, Salehi S, Mohammadzadeh M, Shojaee A, Akbari H, et al. Pre-hospital Trauma Care in Road Traffic Accidents in Kashan, Iran. Arch Trauma Res. 2013; 1 (4): 166-71.

[10] Seid M, Azazh A, Enquselassie F, Yisma E. Injury characteristics and outcome of road traffic accident among victims at Adult Emergency Department of Tikur Anbessa specialized hospital, Addis Ababa, Ethiopia: a prospective hospital-based study. BMC Emergency Medicine. 2015; 15: 10

[11] Huang C. et al. Characteristics and Outcomes of Patients Injured in Road Traffic Crashes and Transported by Emergency Medical Services. Int. J. Environ. Res. Public Health; 2016; 13 (236): doi: 10.3390/ijerph13020236 www.mdpi.com/journal/ijerph.
[12] Gebresenbet RF, Aliyu AD (2019) Injury severity level and associated factors among road traffic accident victims attending the emergency department of Tirunesh Beijing Hospital, Addis Ababa, Ethiopia: A cross-sectional hospitalbased study. PLoS ONE 14 (9): e0222793. https://doi.org/10.1371/journal.pone.0222793.

[13] Bagher A, et al. Analysis of pre-hospital rescue times on mortality in trauma patients in a Scandinavian urban setting. Trauma. 2017; $19 \quad$ (1): 28-34. DOI: $10.1177 / 1460408616649217$ tra.sagepub.com.

[14] Rogers F, Rittenhouse K. The Golden Hour in Trauma: Dogma or Medical Folklore? Journal of Lancaster General Hospital Spring. 2014; 1.9 (1): 1-13.

[15] Newgard C. et al. Revisiting the "Golden Hour": An Evaluation of Out-of-Hospital Time in Shock and Traumatic Brain Injury. Ann Emerg Med. 2016; 66 (1): 30-41 doi: 10.1016/j.annemergmed.2014.12.004.

[16] Dharap SB, Kamath S, Kumar V. Does prehospital time affect the survival of major trauma patients where there is no prehospital care? J Postgrad Med 2017; 63: 169-75.

[17] Post-crash response: Supporting those affected by road traffic crashes. Geneva, World Health Organization, 2016.

[18] Katayama Y, Kitamura T, Kiyohara K, et al. Prehospital factors associated with death on hospital arrival after a traffic crash in Japan: a national observational study. BMJ Open 2019; 9: e025350. doi: 10.1136/BMJopen-2018-025350.

[19] Sultan M, Abebe Y, Welde Tsadik A, Ababa A, Gebre Yesus A, Mould-Millman N. Trends and barriers of emergency medical service use in Addis Ababa; Ethiopia. BMC Emergency $\quad$ Medicine. 2019; 19. https://doi.org/10.1186/s12873-019-0242-5. 